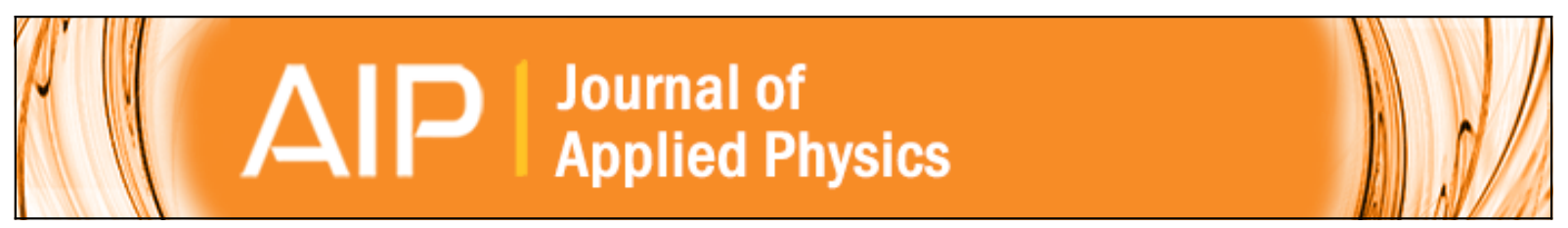

\title{
Rigorous calculation of nonlinear parameters in graphene-comprising waveguides
}

Dimitrios Chatzidimitriou, Alexandros Pitilakis, and Emmanouil E. Kriezis

Citation: Journal of Applied Physics 118, 023105 (2015); doi: 10.1063/1.4926501

View online: http://dx.doi.org/10.1063/1.4926501

View Table of Contents: http://scitation.aip.org/content/aip/journal/jap/118/2?ver=pdfcov

Published by the AIP Publishing

\section{Articles you may be interested in}

Light modulation abilities of nanostructures

AIP Conf. Proc. 1475, 25 (2012); 10.1063/1.4750084

Silicon nanomembrane based photonic crystal waveguide array for wavelength-tunable true-time-delay lines Appl. Phys. Lett. 101, 051101 (2012); 10.1063/1.4739003

$1 \times 12$ Unequally spaced waveguide array for actively tuned optical phased array on a silicon nanomembrane Appl. Phys. Lett. 99, 051104 (2011); 10.1063/1.3619847

Fully complementary metal-oxide-semiconductor compatible nanoplasmonic slot waveguides for silicon electronic photonic integrated circuits

Appl. Phys. Lett. 98, 021107 (2011); 10.1063/1.3537964

Gigahertz photothermal effect in silicon waveguides

Appl. Phys. Lett. 93, 213106 (2008); 10.1063/1.3036957

Frustrated by

old technology?

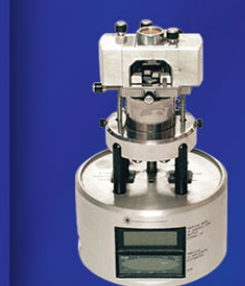

Is your AFM dead

and can't be repaired?

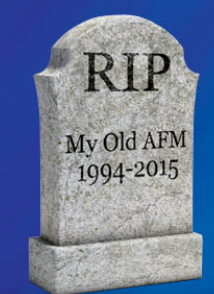

Sick of bad customer support?

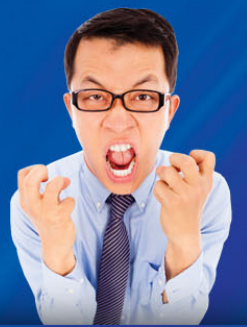

It is time to upgrade your AFM

Minimum $\$ 20,000$ trade-in discount for purchases before August 31st

Asylum Research is today's technology leader in AFM 


\title{
Rigorous calculation of nonlinear parameters in graphene-comprising waveguides
}

\author{
Dimitrios Chatzidimitriou, Alexandros Pitilakis, ${ }^{\text {a) }}$ and Emmanouil E. Kriezis \\ Department of Electrical and Computer Engineering, Aristotle University of Thessaloniki, \\ Thessaloniki GR-54124, Greece
}

(Received 25 March 2015; accepted 29 June 2015; published online 13 July 2015)

\begin{abstract}
We describe a rigorous formalism for the calculation of the nonlinear parameter of arbitrary three-dimensional nanophotonic graphene-comprising waveguides. Graphene is naturally implemented as a zero-thickness conductive sheet, modeled solely by complex linear and nonlinear surface conductivity tensors, whose values are extracted from theoretical models. This representation is compared to the more commonly employed equivalent bulk-medium representation and is found superior. We numerically calculate the nonlinear parameters of several optical waveguide archetypes overlaid with infinite graphene monolayers, including silicon-wire and plasmonic metal-slot and metal-stripe configurations. The metal-slot configuration offers the most promising performance for Kerr-type nonlinear applications. Finally, we apply the same formalism to probe the potential of graphene nanoribbon waveguide nonlinearity in the terahertz band. (C) 2015 AIP Publishing LLC. [http://dx.doi.org/10.1063/1.4926501]
\end{abstract}

\section{INTRODUCTION}

Graphene is a revolutionary two-dimensional material, owing to a combination of unique properties: one-atom thickness, remarkable mechanical strength, and high thermal and electrical conductivity. ${ }^{1,2}$ Naturally, this placed graphene in the forefront of several research fields, including plasmonics ${ }^{3}$ and other guided-wave applications ${ }^{4}$ in the optical spectral region and down to the far-infrared (THz). Of particular interest for photonic and opto-electronic applications is the ability to control graphene's conductivity via tuning its Fermi level, by means of chemical doping or by electrical gating. ${ }^{5}$ Several graphene-comprising optical devices have been theoretically proposed and experimentally demonstrated, including photodetectors, ${ }^{6}$ polarizers, ${ }^{7}$ and modulators. ${ }^{89}$ The nonlinear behavior of graphene has also attracted considerable interest in recent years, after its nonlinearity was theoretically predicted to be rather high. ${ }^{10}$ Graphene-based nonlinear waveguides and their potential applications are intensively investigated, with experimental ${ }^{11,12}$ and theoretical ${ }^{13-15}$ results slowly converging.

The preliminary goal of this work is to institute a framework for the rigorous electromagnetic (Maxwellian) treatment of nanophotonic waveguides that comprise both bulk (3D) and sheet (2D) materials, ${ }^{16}$ of which graphene is a prominent example. We utilize the finite-element method (FEM),${ }^{17}$ which is able to model and seamlessly interface both types of materials, while the vector properties of the electromagnetic fields, as well as the tensor properties of the involved bulk and sheet media, are fully taken into account. Moreover, it will be shown that the sheet representation of graphene is superior to the more commonly employed equivalent (or effective) bulk representation, ${ }^{5}$ both in terms of consistency with underlying physics and of minimized computational effort. The crucial point is the inherent anisotropy exhibited by $2 \mathrm{D}$ materials in $3 \mathrm{D}$ geometries, which is

\footnotetext{
a)alexpiti@auth.gr
}

naturally satisfied in the sheet representation but has to be carefully introduced in the equivalent bulk representation.

The main objective of this work is the rigorous calculation of the nonlinear parameter, $\gamma_{\mathrm{NL}}$, in nanophotonic waveguides comprising bulk and sheet nonlinear media (Fig. 1). It will be shown that $\gamma_{\mathrm{NL}}$, which refers to a given waveguide mode, can be split into contributions from bulk and sheet material nonlinearity. The pulse propagation along such nonlinear waveguides is modeled in terms of the slowly varying envelope approximation and results in a set of coupled propagation equations, commonly referred to as the nonlinear Schrödinger equations (NLSEs), ${ }^{18}$ each one corresponding to a different signal, that is, a different waveguide eigenmode or frequency component. This research area has received renewed attention in the past few years, with the emergence of nanophotonic, plasmonic, and hybrid waveguides ${ }^{19-22}$ breaching the diffraction limit. Our primary focus will be the self-acting phenomena that originate from interaction of guided electromagnetic waves within a narrow frequency band, namely, the optical Kerr effect and the two-photon absorption (TPA). Nevertheless, this formulation can be generalized for other third-order phenomena such as four-wave mixing (FWM), parametric amplification, Raman scattering, and third-harmonic generation (THG). ${ }^{23}$ All these can be modeled via an appropriate third-order nonlinear susceptibility, $\chi^{(3)}$, referring to bulk media. ${ }^{24}$ In this work, we will expand this formalism to include the contribution stemming from sheet materials modeled by a nonlinear conductivity, $\sigma^{(3)}$, whose value is extracted from theoretical models. Finally, the $\gamma_{\mathrm{NL}}$ of graphene-comprising photonic and plasmonic waveguide archetypes will be numerically calculated in order to establish benchmark values and identify optimal designs, both in the optical and THz spectral regions.

This paper is organized as follows: Section II contains the electromagnetic formulation, describing the conductive sheet medium properties, the equivalent bulk medium 


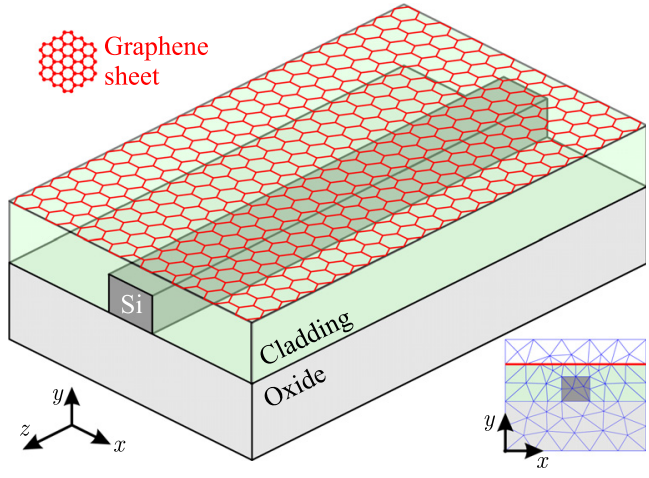

FIG. 1. Silicon wire waveguide overlaid with a low-index cladding and covered by a graphene sheet. In the inset, the FEM-discretized waveguide crosssection in the $x y$-plane.

approximation, and some key aspects of FEM implementation before presenting generalized expressions for the calculation of the nonlinear parameter. Section III briefly reviews theoretical models for the linear and nonlinear conductivity of graphene monolayers and provides a short discussion on the magnitude of its nonlinear response. Section IV deals with the numerical extraction of the nonlinear parameter for typical graphene-comprising waveguide modes and provides insight for their optimization. The paper's conclusions are presented in Section V.

\section{ELECTROMAGNETIC MODELING}

In this section, we describe the electromagnetic representation of graphene and its FEM implementation and, subsequently, present formulas for the rigorous calculation of nonlinear parameters in graphene-comprising waveguides.

\section{A. Graphene as a conductive sheet}

The frequency-domain expression of Maxwell's curl equations is

$$
\begin{gathered}
\nabla \times \tilde{\mathbf{E}}=+i \omega \mu_{0} \bar{\mu}_{\mathrm{r}} \tilde{\mathbf{H}} \\
\nabla \times \tilde{\mathbf{H}}=-i \omega\left(\varepsilon_{0} \tilde{\mathbf{E}}+\tilde{\mathbf{P}}\right)+\tilde{\mathbf{J}}
\end{gathered}
$$

where $\tilde{\mathbf{P}}=\tilde{\mathbf{P}}_{\text {lin }}+\tilde{\mathbf{P}}_{\mathrm{NL}}$ is the electric polarization, $\tilde{\mathbf{J}}=\tilde{\mathbf{J}}_{\text {lin }}$ $+\tilde{\mathbf{J}}_{\mathrm{NL}}$ is the electric current density, and the bar (as in $\bar{\mu}$ ) denotes a tensor in $\mathbb{R}^{3}$. Both $\tilde{\mathbf{P}}$ and $\tilde{\mathbf{J}}$ are separated into a linear and a nonlinear part, the latter considered a perturbation. Equations (1) are derived under the convention $\exp (-i \omega t)$ for the time-harmonic variation.

The linear polarization term can be written as $\tilde{\mathbf{P}}_{\text {lin }}=\varepsilon_{0}$ $\bar{\chi}^{(1)} \tilde{\mathbf{E}}$, where $\bar{\chi}^{(1)}=\bar{\varepsilon}_{\mathrm{r}}-I_{3}$ is a second-rank tensor representing the linear susceptibility. In waveguides comprising only isotropic media, the tensor $\bar{\varepsilon}_{\mathrm{r}}$ degenerates to a scalar value, that is, $\varepsilon_{\mathrm{r}} I_{3}$; similarly, $\bar{\mu}_{\mathrm{r}}=I_{3}$ as optical materials do not exhibit magnetic properties. The nonlinear part of the polarization stems from various physical phenomena and can be modeled with higher-rank susceptibility tensors. ${ }^{25,26}$ In this work, we include only the third-order nonlinear susceptibility, which can be written in the abstract time-domain form $\mathbf{P}_{\mathrm{NL}}=\varepsilon_{0} \bar{\chi}^{(3)} \mid \mathbf{E E E}$, implying a tensor contraction between the fourth-rank tensor $\bar{\chi}^{(3)}$ and three electric field vectors.
For a thorough description of the properties of this susceptibility tensor, see Refs. 18 and 26 .

The electric current density is given by Ohm's law as $\mathbf{J}=\sigma \mathbf{E}$, where $\sigma$ is the conductivity of the medium. The bulk current density can be expressed as a power series in the time-domain fields

$$
\mathbf{J}_{b}=\bar{\sigma}^{(1)}\left|\mathbf{E}+\bar{\sigma}^{(2)}\right| \mathbf{E} \mathbf{E}+\bar{\sigma}^{(3)} \mid \mathbf{E E E}+\cdots,
$$

where $\bar{\sigma}^{(n)}$ are complex-valued $(n+1)$-rank conductivity tensors (in units of $\left[(\mathrm{S} / \mathrm{m})(\mathrm{m} / \mathrm{V})^{n-1}\right]$ ) analogous to the susceptibility tensors $\bar{\chi}^{(n)}$. Converted to the frequency domain, the first term of Eq. (2) represents the linear current density, $\tilde{\mathbf{J}}_{\text {lin }}$, and the following terms represent the nonlinear current density, $\tilde{\mathbf{J}}_{\mathrm{NL}}$. Without loss of generality, bulk-medium conductivity can be introduced as an imaginary part to the susceptibility tensor of respective rank, and thus $\mathbf{J}_{b}$ is absorbed by $\mathbf{P}$ in Eq. (1b). However, in the presence of conductive sheets of infinitesimally small (practically zero) thickness, the overall current density $\mathbf{J}$ also involves sheet current density contributions, $\mathbf{J}_{s}$, which are naturally expressed by an expression analogous to Eq. (2)

$$
\mathbf{J}_{s}=\bar{\sigma}_{s}^{(1)}\left|\mathbf{E}+\bar{\sigma}_{s}^{(2)}\right| \mathbf{E E}+\bar{\sigma}_{s}^{(3)} \mid \mathbf{E E E}+\cdots
$$

with $\bar{\sigma}_{s}^{(n)}$ being surface conductivity tensors, measured in $\left[\mathrm{S}(\mathrm{m} / \mathrm{V})^{\mathrm{n}-1}\right]$. The overall current density can be written as $\mathbf{J}=\mathbf{J}_{b}+\mathbf{J}_{s} \delta_{s}(\mathbf{r})$, where $\delta_{s}$ is a surface Dirac function. ${ }^{27}$

The 2D nature of sheet materials implies an inherent anisotropy in vectorial $\mathbb{R}^{3}$ formulations. Specifically, the surface current $\tilde{\mathbf{J}}_{s}$ in graphene can only have components tangential to the sheet and, in the linear case, does not implicate the normal electric field component. Assuming a sheet normal to the $y$-axis, this type of anisotropy is modeled by the second-rank surface conductivity tensor

$$
\bar{\sigma}_{\mathrm{s}}^{(1)}=\left[\begin{array}{ccc}
\sigma_{s, x x} & 0 & \sigma_{s, x z} \\
0 & 0 & 0 \\
\sigma_{s, z x} & 0 & \sigma_{s, z z}
\end{array}\right],
$$

containing four independent parameters. However, for most practical applications, graphene exhibits a number of symmetries that simplify Eq. (4), specifically $\sigma_{s, x z}=\sigma_{s, z x} \approx 0$ (except under the presence of strong magnetic field ${ }^{28}$ ) and $\sigma_{s, x x} \equiv \sigma_{s, z z}=\sigma_{c}$. In this case, graphene can be modeled by a single surface conductivity complex value $\left(\sigma_{c}\right)$ and knowledge of the normal vector (n) to derive the surface current

$$
\tilde{\mathbf{J}}_{s}=\bar{\sigma}_{s}^{(1)} \tilde{\mathbf{E}}=\sigma_{c} \tilde{\mathbf{E}}_{\|}=\sigma_{c}[\mathbf{n} \times(\tilde{\mathbf{E}} \times \mathbf{n})],
$$

where $\tilde{\mathbf{E}}_{\|}$are the electric field components tangential to the sheet. For inhomogeneous (or non-planar) graphene sheets, the value of $\sigma_{c}$ (or $\mathbf{n}$ ) can vary in space.

Returning to the higher-order terms of Eqs. (2) and (3) responsible for the nonlinear current density $\tilde{\mathbf{J}}_{\mathrm{NL}}$, we will focus exclusively on the third-order nonlinear surface conductivity $\bar{\sigma}_{s}^{(3)}$. Even-order processes, such as secondharmonic generation (SHG), in unstrained graphene are 
vanishingly small due to graphene's unit cell inversion symmetry and can become appreciable only when the sheet is deposited on a substrate, ${ }^{23}$ is deformed/strained, ${ }^{29}$ or by using DC current to generate an asymmetric steady state of the electron density. ${ }^{30}$ In any case, this work focuses on selfacting nonlinear phenomena, namely, the Kerr effect and TPA, that can be described by a nonlinear surface current density with the following general expression for its $j$-th cartesian component ${ }^{26,31}$

$$
J_{s, j, \mathrm{NL}}=\mathbf{J}_{s, \mathrm{NL}} \cdot \mathbf{j}=\frac{3}{4} \sum_{k l m} \sigma_{s, j k l m}^{(3)} E_{k} E_{l}^{*} E_{m},
$$

where the subscripts $\{j, k, l, m\}$ denote cartesian components, that is, $\{x, y, z\}$, and $\sigma_{s, j k l m}^{(3)}$ are the 81 complex-valued elements of the fourth-rank tensor $\bar{\sigma}_{s}^{(3)}$. The form of $\bar{\sigma}_{s}^{(3)}$ for graphene can be derived from the hexagonal symmetry group $D_{6 h}(6 / \mathrm{mmm}) .{ }^{14,23}$ When deposited on a substrate, graphene loses its inversion symmetry along the surface normal, thus giving rise to $C_{6 v}(6 \mathrm{~mm})$ symmetry. ${ }^{23}$ Both these symmetry groups typically refer to bulk/3D media and allow for 21 nonzero (10 independent) elements when $\{x, y, z\}$ match the crystal coordinate system. In sheet/2D media, the normal component of $\mathbf{J}_{s, \mathrm{NL}}$ is required to vanish, and, as a result, the "reduced" $2 \mathrm{D}$ form of the $D_{6 h}$ or $C_{6 v}$ group allows for 14 nonzero ( 6 independent) elements. Furthermore, using all possible symmetries to reduce the number of elements leads to a $\bar{\sigma}_{s}^{(3)}$ tensor that entails 8 nonzero elements that all depend on a single complex nonlinearity value $\left(\sigma_{3}\right)$, according to

$$
\sigma_{s, j k l m}^{(3)}=\sigma_{3} \frac{1}{3}\left(\delta_{j k} \delta_{l m}+\delta_{j m} \delta_{k l}+\delta_{j l} \delta_{m k}\right)
$$

In this expression, $\delta_{p q}$ is the Kronecker delta and $\{j, k, l, m\}$ correspond only to the two cartesian components that are tangential to the graphene sheet. For instance, when the sheet is normal to the $y$-axis, then $\sigma_{s, x x x x}^{(3)} \equiv \sigma_{s, z z z z}^{(3)}=\sigma_{3}$ and $\sigma_{s, m m n n}^{(3)} \equiv \sigma_{s, \text { mnтn }}^{(3)} \equiv \sigma_{s, m n n m}^{(3)}=\sigma_{3} / 3$, where $\{m, n\}=\{x, z\}$. The tensor of Eq. (7) is an overly simplified version of $D_{6 h}$ or $C_{6 v}$ and describes a sheet material that can be considered as the 2D-equivalent of an isotropic centrosymmetric bulk/ 3D medium; in the latter case, subscripts $\{j, k, l, m\}$ would scan all three cartesian components and the fourth-rank tensor would comprise 21 nonzero (1 independent) elements.

\section{B. Equivalent bulk medium representation}

The sheet representation of graphene is its most natural macroscopic modeling in the framework of Maxwellian electromagnetism. Nevertheless, another representation is, as of now, more commonly used in the literature: the equivalent bulk medium approach, where graphene is modeled as a bulk-material layer of sub-nanometer (nonzero) thickness. ${ }^{5}$ The bulk representation of graphene is useful when a comparison with other bulk materials is required and can be easily introduced in any formulation designed for bulk materials. However, it has two drawbacks: First, it requires careful consideration of the anisotropy of the original sheet material and, second, it increases the computational burden, as will be discussed in Sec. II C.

The properties of this equivalent bulk material, namely, the refractive index $\left(n_{0, \text { eq }}\right)$ and the nonlinear index $\left(n_{2, \text { eq }}\right)$, are derived from the sheet material properties, that is, the set of complex conductivity tensors: A sheet material of the surface conductivity tensor $\bar{\sigma}_{s}^{(m)}$ can be represented by an equivalent bulk medium of thickness $d$ and susceptibility tensor $\bar{\chi}_{\text {eq }}^{(m)}=i \bar{\sigma}_{s}^{(m)} /\left(\omega \varepsilon_{0} d\right)$, according to Eq. (1b). Consequently, the equivalent relative permittivity for a graphene sheet with $d_{\mathrm{gr}} \approx 0.34 \mathrm{~nm}$ can be evaluated as

$$
\varepsilon_{\mathrm{r}, \mathrm{eq}}=1+\chi_{\mathrm{eq}}^{(1)}=1+\frac{i \sigma_{c}}{\omega \varepsilon_{0} d_{\mathrm{gr}}},
$$

where $\sigma_{c}$ (in units of Siemens) is the linear scalar surface conductivity, as in Eq. (5). The scalar equivalent refractive index of graphene is given by $n_{0, \text { eq }}=\sqrt{\varepsilon_{\mathrm{r}, \text { eq }}}$. The tensor representation has been dropped in Eq. (8) denoting an isotropic material. Similarly, the equivalent nonlinear index $n_{2, \text { eq }}$ (in units of $\mathrm{m}^{2} / \mathrm{W}$ ) of a graphene sheet can be evaluated according to Refs. 25 and 26 as

$$
n_{2, \mathrm{eq}}=\frac{3}{4} \frac{\chi_{\mathrm{eq}}^{(3)}}{\varepsilon_{\mathrm{r}, \mathrm{eq}}} Z_{0}=\frac{3}{4} \frac{i \sigma_{3} /\left(\omega \varepsilon_{0} d_{\mathrm{gr}}\right)}{1+i \sigma_{c} /\left(\omega \varepsilon_{0} d_{\mathrm{gr}}\right)} Z_{0},
$$

where $Z_{0}=1 /\left(\varepsilon_{0} c_{0}\right) \approx 377 \Omega$ is the free space impendence and $\sigma_{3}$ [in units of $\mathrm{S}(\mathrm{m} / \mathrm{V})^{2}$ ] is the scalar nonlinear surface conductivity (Eq. (7)). Note that the sign of $\operatorname{Re}\left\{n_{2, \text { eq }}\right\}$ can change according to the angle of the complex number $\varepsilon_{\mathrm{r}, \mathrm{eq}}$ : assuming a purely imaginary $\sigma_{3}$, a sign-transition for $\operatorname{Re}\left\{n_{2, \text { eq }}\right\}$ occurs for $\left|\operatorname{Re}\left\{n_{0, \text { eq }}\right\}\right| \equiv\left|\operatorname{Im}\left\{n_{0, \text { eq }}\right\}\right|$. Nevertheless, the real part of the nonlinear parameter $\left(\gamma_{\mathrm{NL}}\right)$ eventually controls the sign and magnitude of the accumulated phase. ${ }^{18}$

The isotropic bulk representation of graphene is sufficient for a limited range of practical applications. However, this oversimplified approach fails to fully capture the inherent anisotropy of sheet media in $\mathbb{R}^{3}$ and is found wanting in more demanding problems. To amend this issue, the equivalent bulk medium should be rendered anisotropic, with respect to its linear and nonlinear susceptibility. In this regard, any anisotropy contained in the linear and nonlinear surface conductivity tensors should be inherited by the respective susceptibility tensors. For instance, the anisotropic $\bar{\varepsilon}_{\text {r,eq }}$ tensor will stem from the second-rank $\bar{\sigma}_{s}^{(1)}$ tensor of Eq. (4) as

$$
\bar{\varepsilon}_{\mathrm{r}, \mathrm{eq}}=\left[\begin{array}{ccc}
\varepsilon_{\mathrm{r}, \mathrm{eq}} & 0 & 0 \\
0 & 1 & 0 \\
0 & 0 & \varepsilon_{\mathrm{r}, \mathrm{eq}}
\end{array}\right],
$$

excluding the off-diagonal terms. Similarly, the nonlinear fourth-rank susceptibility tensor $\bar{\chi}_{\text {eq }}^{(3)}$ will stem from the respective surface conductivity tensor of Eq. (7), inheriting its 8 nonzero elements that will only depend on $\sigma_{3}$ or, equivalently, on $n_{2, \text { eq }}$ and $\varepsilon_{\mathrm{r}, \mathrm{eq}}$. Note that, for regular isotropic bulk materials, $\bar{\chi}^{(3)}$ has a total of 21 nonzero elements that depend on $\chi^{(3)}$ or on both $n_{2}$ and $\varepsilon_{\mathrm{r}}{ }^{20}$ Finally, these anisotropic tensors will have to be rotated when the sheet does not have a 
trivial orientation, as is the case of Eq. (10) where the sheet was normal to a principal axis; expressions for these rotations will be presented in Section II C.

Concluding, sheet or sheet-like (very thin) materials are naturally modeled by a set of surface conductivities, $\sigma_{c}$ and $\sigma_{3}$, respectively. However, these materials can also be modeled by an equivalent set of bulk-medium properties $\left(\varepsilon_{\mathrm{r}, \mathrm{eq}}\right.$ and $n_{2, \text { eq }}$ ), Eqs. (8)-(9), which should be cast in tensor form so that they additionally satisfy the inherent anisotropy of sheet materials. The overruling parameter is the artificial thickness attributed to the sheet or sheet-like material, which is assumed to be very small compared to the operating wavelength and the waveguide dimensions, as is the case for graphene.

\section{Finite element method}

The FEM ${ }^{17}$ has been established as the definitive numerical tool for modeling arbitrary cross-sectional nanophotonic waveguides in the past decade. Graphene deviates from typically used bulk materials in the sense that its thickness is negligible when compared to the wavelength or the size of the waveguide features. Nevertheless, such two-dimensional materials can be elegantly introduced in the FEM by means of an electric surface conductivity and attributed to the interface between two media that is occupied by the sheet. ${ }^{17}$ In geometric terms, if a bulk material is modeled by a finite surface (or volume), then a sheet material is modeled by the edges (or faces) of the element, respectively. In our FEM modeling, bulk materials are modeled by a linear and a nonlinear susceptibility tensor, $\bar{\chi}^{(1)}$ and $\bar{\chi}^{(3)}$, respectively. Similarly, sheet materials such as graphene are modeled by a linear and a nonlinear surface conductivity tensor, $\bar{\sigma}_{s}^{(1)}$ and $\bar{\sigma}_{s}^{(3)}$, respectively. All elements of these tensors are complex valued, and they can have frequency dispersion and/or inhomogeneous distribution.

Graphene can alternatively be modeled as an equivalent bulk-medium layer of sub-nanometer thickness (Section II B). However, discretizing very thin layers leads to a large number of degrees of freedom (unknowns), even for the versatile FEM, and hence to increased computation times. In 2D geometries, that is, waveguide cross-sections, this extra burden can be tolerable but it becomes excessive in $3 \mathrm{D}$ structures; this further justifies the use of the sheet representation for graphene.

The interface conditions for the electric and magnetic fields between two bulk media sandwiching a conductive sheet take the form

$$
\begin{array}{r}
\mathbf{n} \times\left(\tilde{\mathbf{E}}_{2}-\tilde{\mathbf{E}}_{1}\right)=0, \\
\mathbf{n} \times\left(\tilde{\mathbf{H}}_{2}-\tilde{\mathbf{H}}_{1}\right)=\tilde{\mathbf{J}}_{s},
\end{array}
$$

where $\mathbf{n}$ is the vector normal to the interface, with direction from medium 1 to medium 2, and the surface current density $\tilde{\mathbf{J}}_{s}$ is subject to Ohm's law. The discontinuity of the tangential H-fields, Eq. (11b), is proportional to the surface conductivity of the sheet, vanishing in its absence.

Employing the Galerkin FEM technique ${ }^{17}$ for the linear regime, we form the vector wave equation by taking the curl of Eq. (1a) and replacing it in Eq. (1b). We then take the dot product with an appropriate weighting function $\tilde{\mathbf{E}}_{a}$ and integrate over the entire volume of the problem $V$. Using integration by parts, assuming that the fields vanish on the enclosing boundary of $V$ and, finally, employing the surface current as $\tilde{\mathbf{J}}_{s} \delta_{s}(\mathbf{r})$, we derive

$$
\begin{aligned}
& \iiint_{V}\left\{\left(\nabla \times \tilde{\mathbf{E}}_{a}\right) \cdot\left[\bar{\mu}_{r}^{-1}(\nabla \times \tilde{\mathbf{E}})\right]-k_{0}^{2} \tilde{\mathbf{E}}_{a} \cdot\left[\bar{\varepsilon}_{\mathrm{r}} \tilde{\mathbf{E}}\right]\right\} \mathrm{d} V \\
& \quad=i \omega \mu_{0} \iint_{S} \tilde{\mathbf{E}}_{a} \cdot\left[\bar{\sigma}_{s}^{(1)} \tilde{\mathbf{E}}\right] \mathrm{d} S,
\end{aligned}
$$

where the LHS is the standard FEM modeling of bulk media, whereas the RHS term models conductive sheets and vanishes in their absence. Considering the simplest form of a conductive sheet (Eq. (5)), the integral in the RHS term of Eq. (12) can be cast in the form

$$
\iint_{S} \tilde{\mathbf{E}}_{a} \cdot\left[\bar{\sigma}_{s}^{(1)} \tilde{\mathbf{E}}\right] \mathrm{d} S=\sigma_{c} \iint_{S}\left(\mathbf{n} \times \tilde{\mathbf{E}}_{a}\right) \cdot(\mathbf{n} \times \tilde{\mathbf{E}}) \mathrm{d} S,
$$

where all the information of the tensor $\bar{\sigma}_{s}^{(1)}$ is now contained in $\sigma_{c}$ and $\mathbf{n}$, the scalar surface conductivity of graphene and the normal vector, respectively.

When the form of linear conductivity does not permit the above simplification, then the second-rank tensor $\bar{\sigma}_{s}^{(1)}$ appearing in Eq. (12) is computed by an appropriate rotation from a reference orientation, $\bar{\sigma}_{s, \text { ref }}^{(1)}$ [as, e.g., in Eq. (4)], using the matrix multiplication formula

$$
\bar{\sigma}_{s}^{(1)}=R \bar{\sigma}_{s, \text { ref }}^{(1)} R^{T},
$$

where the superscript $T$ denotes a matrix transpose. The rotation matrix $R$ is a real $3 \times 3$ array whose values are direction cosines, defined by the angles between the corresponding axes of the reference and the rotated coordinate systems. For the rotation of the nonlinear fourth-rank conductivity tensor, a generalized expression ${ }^{26}$ is employed

$$
\sigma_{s, j k l m}^{(3)}=\sum_{a b c d}^{x y z} R_{j a} R_{k b} R_{l c} R_{m d} \sigma_{s, a b c d, \text { ref }}^{(3)},
$$

involving summation over the indices $\{a, b, c, d\}$ that correspond to the cartesian axes $\{x, y, z\} . R_{p q}$ is a scalar value, referring to the $p q$-element of the rotation matrix $R$. Equations (14) and (15) equally hold for the linear and nonlinear susceptibility tensors, $\bar{\chi}^{(n)}$, with $n=1$ and 3, respectively.

Equation (12) can be used for full 3D-FEM modeling, but in this work, we cast it in a 2D form, appropriate for waveguide eigenmode analysis, assuming a longitudinally invariant waveguide (Fig. 1). To this end, we can readily substitute the volume and surface integrals with the surface and line integrals, respectively, since the space of the problem is now the waveguide cross-section in the $x y$-plane. For the spectral eigenmode analysis at a specified frequency, the electric field is written as $\tilde{\mathbf{E}}=\mathbf{e}(x, y) \exp \left(+i n_{\mathrm{eff}} k_{0} z\right)$, where $\mathbf{e}(x, y)$ is the transverse spectral envelope of a guided mode and $n_{\text {eff }}$ is its complex valued effective index. The results are the transverse envelopes (eigenvectors) and effective indices 
(eigenvalues) of all guided modes of the waveguide. Each mode's propagation losses can be quantified by the propagation length, defined as the distance where the power drops to $1 / e(-4.34 \mathrm{~dB})$ and calculated by the imaginary part of the effective index as $L_{\text {prop }}=\lambda /\left(4 \pi \operatorname{Im}\left\{n_{\text {eff }}\right\}\right)$.

\section{Nonlinear waveguide parameters}

In this section, we outline a NLSE formalism for the study of nonlinear phenomena that originate from interaction of guided electromagnetic waves in a narrow frequency band (e.g., Kerr effect, TPA, or FWM). ${ }^{20-22}$ Nonlinear propagation is tackled by introducing the nonlinear polarization $\left(\mathbf{P}_{\mathrm{NL}}\right)$ and current $\left(\mathbf{J}_{\mathrm{NL}}\right)$ as a perturbation to the linear solution: We extract waveguide modes by means of linear eigenmode analysis of the waveguide cross-section and use that information to calculate the parameters of our NLSE model. We focus on the calculation of the nonlinear parameter, $\gamma_{\mathrm{NL}}$ in units of $\left[\mathrm{m}^{-1} \mathrm{~W}^{-1}\right]$, that quantifies the nonlinearityinduced phase shift and loss of each mode. This parameter, along with the propagation length $L_{\text {prop}}$, is used for the mode's figure-of-merit (FoM) as $\mathcal{F}=\gamma_{\mathrm{NL}} L_{\text {prop }}$, in units of $\mathrm{W}^{-1}$. It can be shown that the peak optical power required for the manifestation of a Kerr-type nonlinear effect is inversely proportional to $\mathcal{F},{ }^{22}$ while an extra constraint for nanophotonic circuits is footprint miniaturization. Overall, graphene nanophotonic waveguide technology can be used to satisfy at least two of these criteria: high nonlinear parameter, low losses, and small dimensions.

Standard NLSE techniques are used to calculate $\gamma_{\mathrm{NL}}$ for a specific mode of a given waveguide. ${ }^{20,21}$ Returning to Eq. (1b), we separate the linear and nonlinear polarization and current and recast the equation in the following form:

$$
\nabla \times \tilde{\mathbf{H}}=-i \omega \varepsilon_{0}\left(\overline{\varepsilon_{\mathrm{r}}}-\frac{\bar{\sigma}^{(1)}}{i \omega \varepsilon_{0}}\right) \tilde{\mathbf{E}}-i \omega\left(\tilde{\mathbf{P}}_{\mathrm{NL}}+\frac{i}{\omega} \tilde{\mathbf{J}}_{\mathrm{NL}}\right)
$$

Comparing Eq. (16) to Eq. (5) of Ref. 21 or Eq. (3) of Ref. 20, we note that the nonlinear current can be added to the nonlinear polarization forming the overall perturbation term $\tilde{\mathbf{P}}_{\mathrm{NL}}^{\prime}(\omega)=\tilde{\mathbf{P}}_{\mathrm{NL}}+i \omega^{-1} \tilde{\mathbf{J}}_{\mathrm{NL}}$. It can be inferred that $\gamma_{\mathrm{NL}}$ can eventually be split into two parts, one resulting from the mode-profile overlapping with nonlinear bulk media $\left(\gamma_{b}\right)$ and one from overlapping with nonlinear sheet media $\left(\gamma_{s}\right)$, which is the case of graphene.

Assuming a single-mode waveguide for simplicity and following the procedure described in Ref. 21, we write the electric field as $\tilde{\mathbf{E}}(\mathbf{r}, \omega)=\tilde{A}\left(z, \omega-\omega_{0}\right) \mathbf{e}\left(x, y, \omega_{0}\right) \exp \left(+i \beta_{0} z\right) /$ $\sqrt{N}$, where $\tilde{A}$ is the slowly varying spectral field envelope, $\omega_{0}$ is the operating frequency, $\mathbf{e}$ is the transverse envelope profile, and $\beta_{0}$ is the mode propagation constant at $\omega_{0}$. The normalization constant $N$ is measured in Watt, so that $|\tilde{A}|^{2}$ represents power, and is given by $N=0.5\left|\iint\left(\mathbf{e} \times \mathbf{h}^{*}\right) \cdot \mathbf{z d} S\right|$; the integral is calculated over the waveguide cross-section, while the integrand corresponds to the time-averaged guided power density, derived from the Poynting vector. The definition of $N$ is related to the mode orthogonality ${ }^{32}$ and holds for lossless and lossy waveguides alike. ${ }^{22}$ Also, in other formulations, the constant $N$ is defined as the real part of the integral, instead of its absolute value, and omits the 0.5 factor, ${ }^{20}$ that is, $N^{\prime}=\operatorname{Re}\left\{\iint\left(\mathbf{e} \times \mathbf{h}^{*}\right) \cdot \mathbf{z d} S\right\} \approx 2 N$; the two variations are practically equivalent. The time-domain expression for the nonlinear propagation equation in a single-mode waveguide is given by

$$
\frac{\partial A}{\partial z}=\frac{i \omega_{0} e^{i\left(\omega_{0} t-\beta_{0} z\right)}}{2 \sqrt{4 N}} \iint \mathbf{e}^{*} \cdot\left(\mathbf{P}_{\mathrm{NL}}+\frac{i}{\omega_{0}} \mathbf{J}_{\mathrm{NL}}\right) \mathrm{d} S+\mathcal{L} A,
$$

where $A(z, t)$ is the Inverse Fourier Transform of the spectral envelope $\tilde{A}\left(z, \omega-\omega_{0}\right)$ and the term in parentheses in the RHS corresponds to the modified nonlinear polarization $\mathbf{P}_{\mathrm{NL}}^{\prime}$, assuming that $\mathbf{J}_{\mathrm{NL}}$ is constant over the spectral region near $\omega_{0}$. Linear contributions to Eq. (17), namely, frequency dispersion and propagation losses, are contained in the operator $\mathcal{L}$. Finally, the extension to multimode propagation in nonlinear waveguides is straightforward. ${ }^{20-22}$

Replacing the expressions of the nonlinear polarization $\mathbf{P}_{\mathrm{NL}}$ and conductivity $\mathbf{J}_{\mathrm{NL}}$ in Eq. (17), we arrive at the following expression for the Continuous Wave regime:

$$
\frac{\partial A}{\partial z}=i\left(\gamma_{b}+\gamma_{s}\right)|A|^{2} A-\frac{1}{2 L_{\text {prop }}} A
$$

where the nonlinear parameters are defined as

$$
\begin{aligned}
& \gamma_{b}=\frac{3 \omega_{0} \epsilon_{0}}{4(2 N)^{2}} \sum_{j k l m}^{x y z} \iint \chi_{j k l m}^{(3)} e_{j}^{*} e_{k} e_{l}^{*} e_{m} \mathrm{~d} S, \\
& \gamma_{s}=i \frac{3}{4(2 N)^{2}} \sum_{j k l m}^{x y z} \int \sigma_{s, j k l m}^{(3)} e_{j}^{*} e_{k} e_{l}^{*} e_{m} \mathrm{~d} \ell,
\end{aligned}
$$

originating from the bulk and sheet material nonlinearity, respectively. Note how the imaginary part of $\bar{\sigma}_{s}^{(3)}$ is responsible for the sheet-induced nonlinear phase shift and that the integration is now carried over the graphene sheet instead of the waveguide cross-section. Furthermore, the nonlinear absorption processes are introduced through the real part of $\bar{\sigma}_{s}^{(3)}$, similarly to the inclusion of the TPA parameter as an imaginary part in the nonlinear index $n_{2}$ of a bulk medium. ${ }^{20}$

Assuming the simplest forms of $\bar{\chi}^{(3)}$ [e.g., Eq. (14) of Ref. 20, for $\rho=1]$ and $\bar{\sigma}_{s}^{(3)}$ [Eq. (7)], the tensor contraction operations implied by the quad summation in Eqs. (19) can be simplified to

$$
\begin{aligned}
& \gamma_{b}=\frac{\omega_{0} \epsilon_{0}}{(2 N)^{2}} \iint \chi_{3}\left(\frac{1}{2}|\mathbf{e}|^{4}+\frac{1}{4}|\mathbf{e} \cdot \mathbf{e}|^{2}\right) \mathrm{d} S, \\
& \gamma_{s}=i \frac{1}{(2 N)^{2}} \int \sigma_{3}\left(\frac{1}{2}\left|\mathbf{e}_{\|}\right|^{4}+\frac{1}{4}\left|\mathbf{e}_{\|} \cdot \mathbf{e}_{\|}\right|^{2}\right) \mathrm{d} \ell,
\end{aligned}
$$

respectively, where $\mathbf{e}_{\|}$is the electric field component of the transverse envelope that is tangential to the conductive sheet. $\chi_{3}(x, y)$ and $\sigma_{3}(x, y)$ are scalar complex values for the nonlinear susceptibility and surface conductivity, respectively, that can be inhomogeneous in the waveguide cross-section.

Equations (19) and (20) not only provide a rigorous method for the evaluation of the nonlinear parameters but 
also enable the comparison of the bulk and sheet nonlinearity contributions. In this way, the comparison is made between meaningful parameters referring to waveguide modes and not between intermediate quantities such as material properties or geometric attributes. The nonlinear parameter for sheet materials emerges naturally through the current density (Eq. (17)), thus extending a framework that conceptually refers to waveguides comprising only bulk materials. Moreover, Eq. (17) is general in the sense that, in theory, it can model any nonlinear phenomena as long as we have knowledge of their nonlinear polarization and current. The only approximations made up to this point are that the nonlinear terms are small perturbations to the respective linear terms and that they have an instantaneous response. Consequently, a similar perturbation approach can also be used to model nonlinear guided-wave phenomena involving vastly different frequency bands, for example, $\mathrm{THG}^{14,23}$ or SHG. ${ }^{33}$ In these cases, phase-matching between the modes at different frequency bands must be satisfied and, specifically for SHG, this is usually accomplished using some sort of periodic spatial alteration of the waveguide along the propagation direction.

\section{PHYSICAL PROPERTIES}

In this section, we will briefly discuss the physical properties of graphene monolayers and provide a discussion on the magnitude of graphene nonlinearity.

\section{A. Linear conductivity}

The linear part of the surface conductivity $\bar{\sigma}_{s}^{(1)}$, in its simplest scalar form as $\sigma_{c}$ in Eq. (5), consists of contributions from intraband and interband mechanisms for the absorption of photons of energy $\mathcal{E}_{\mathrm{ph}}=\hbar \omega$ by a graphene monolayer/sheet. The overall scalar linear surface conductivity is $\sigma_{c}=\sigma_{c \text {,intra }}+\sigma_{c \text {,inter }}$ (in Siemens) and has a real part and an imaginary part, which affect the losses and phase velocity of waves propagating in its vicinity, respectively. The conductivity of graphene can be tuned via chemical doping or by external electrostatic biasing; both these effects are assumed enclosed within a single parameter, the chemical potential $\left(\mu_{c}\right)$, which quantifies the Fermi level difference with respect to the Dirac point. This parameter typically ranges from zero, which corresponds to undoped (pristine) and unbiased graphene, up to $\pm 1 \mathrm{eV} .{ }^{34}$ Finally, under the presence of external magnetic biasing, graphene can exhibit anisotropic linear conductivity, described by $\sigma_{s, x z}=-\sigma_{s, z x} \neq$ 0 in Eq. (4) and referred to as the local Hall effect regime ${ }^{28,35}$ this configuration does not come into the present study.

The intraband contribution term is given by the Drudelike expression ${ }^{34,36}$

$$
\sigma_{c, \text { intra }}=i \frac{e^{2} \mu_{c}}{\pi \hbar^{2}\left(\omega+i / \tau_{1}\right)} \times \mathcal{T}\left(\frac{\mu_{c}}{2 k_{B} T}\right),
$$

where $\mathcal{T}(x)=x^{-1} \ln [2 \cosh (x)], T$ is the absolute temperature, and $\tau_{1}$ is the relaxation time for intraband absorption, which in a phenomenological way takes into account losses due to electron impurity, electron defect, and electronphonon scattering. ${ }^{37}$ This relaxation time is sometimes expressed in energy or $\mathrm{eV}$ units as $\Gamma=\hbar /(2 \tau) \cdot{ }^{34}$ In the optical regime, $\tau_{1} \approx 10 \mathrm{fs},{ }^{38}$ whereas values in the range of 40 ps have been deemed feasible for the $\mathrm{THz}$ regime at room temperature. ${ }^{39}$ In practical configurations, the temperaturedependent term can be dropped from Eq. (21) as $\mathcal{T} \rightarrow 1$ for $x>1$, that is, when $\mu_{c}>50 \mathrm{meV}$ for $T=300 \mathrm{~K}$.

The interband contribution term has a more complicated dependence on $\mu_{c}$, capturing the physical properties of graphene at the transition point $\mu_{c} \approx 0.5 \hbar \omega$. Specifically, for $\mu_{c}>0.5 \hbar \omega$, the interband absorption mechanism vanishes and so does the real part of the interband conductivity term, whereas a negative peaking of the imaginary part is observed at $\mu_{c}=0.5 \hbar \omega$ (Fig. 2(a)). This term is negligible in the THz region, except for the ideal configuration of pristine graphene. An expression for the interband term in the optical regime is extracted in Ref. 34

$$
\sigma_{c, \text { inter }}=i \frac{e^{2}}{4 \pi \hbar} \ln \left[\frac{2\left|\mu_{c}\right|-\hbar\left(\omega+i / \tau_{2}\right)}{2\left|\mu_{c}\right|+\hbar\left(\omega+i / \tau_{2}\right)}\right],
$$

where $\tau_{2} \approx 1.2 \mathrm{ps}$ is the relaxation time for interband absorption. ${ }^{38}$ Equation (22) holds for $\hbar \omega, \mu_{c} \gg k_{B} T$, that is, neglecting the effect of temperature, an approximation that is valid at optical frequencies, $T \approx 300 \mathrm{~K}$ and $\mu_{c}>0.1 \mathrm{eV}$. Expressions taking into account the temperature dependence of the interband term can be found in Refs. 35 and 36.

The equivalent bulk medium parameters of graphene (Eq. (8)) as a function of $\mu_{c}$ are also depicted in Fig. 2. Note that a negative $\operatorname{Re}\left\{\varepsilon_{\mathrm{r}}\right\}$ of large magnitude is the necessary condition for highly confined TM polarized Surface Plasmon Polariton (SPP) waves propagating along the interface of graphene with a dielectric medium. Specifically, for a guided plasmonic mode at the interface of a metal $\left(\varepsilon_{m}\right)$ with a dielectric $\left(\varepsilon_{d}\right)$, it is required that $\operatorname{Re}\left\{\varepsilon_{m}\right\}<0$ and $\left|\varepsilon_{m}\right| \gg\left|\varepsilon_{d}\right|$. For graphene, this plasmonic regime requires a positive imaginary part in the linear conductivity $\sigma_{c}$, assuming the $e^{-i \omega t}$ convention. This behavior is especially pronounced in lower frequencies, that is, in the $\mathrm{THz}$ regime, ${ }^{28}$ leading to extremely high confinement and effective mode indices at the expense of losses. In the optical regime, it is negligible and becomes barely appreciable at very high $\mu_{c}$, for example, in the right side of Fig. 2(b).
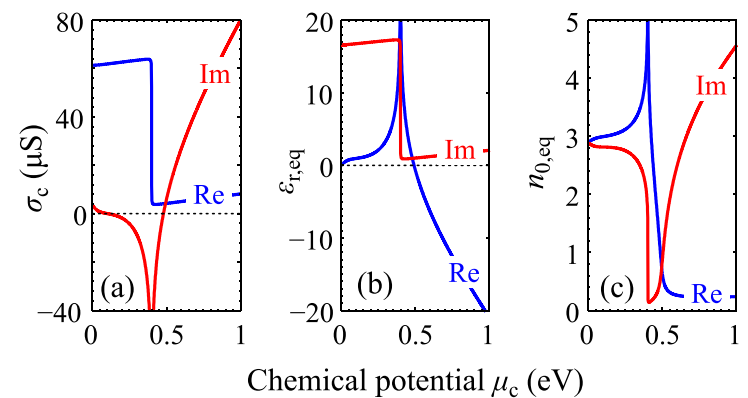

FIG. 2. Real and imaginary parts of graphene: (a) overall surface conductivity, (b) equivalent relative permittivity, (c) equivalent refractive index, as a function of chemical potential $\mu_{c}$ at $\lambda=1550 \mathrm{~nm}$. All curves are symmetric for negative $\mu_{c}$. 


\section{B. Nonlinear conductivity}

The nonlinear conductivity of graphene in the optical regime has been a subject of much debate over the last years, ever since the first experimental evidence of remarkably large values. ${ }^{10}$ However, the extracted values for the magnitude of nonlinear effects in graphene show discrepancies and strongly depend on measurement method, frequency, and sample preparation; the reader is referred to the introduction of Ref. 14 for a review of the pertinent state of the art. In this work, we will briefly present the existing theoretical models for graphene's nonlinearity to provide an estimation of its magnitude.

The magnitude of the Kerr effect, used for all-optical signal processing, ${ }^{15}$ is proportional to the imaginary part of the nonlinear surface conductivity. For lack of experimental evidence, TPA-related phenomena are not discussed here, but they can be easily introduced as a real part to the nonlinear conductivity. Assuming the simplest form of the fourthrank tensor $\bar{\sigma}_{s}^{(3)}$ (Eq. (7)), the corresponding scalar nonlinear surface conductivity in the optical regime can be approximated by ${ }^{10}$

$$
\sigma_{3, \mathrm{opt}}=-i \frac{9 e^{4} v_{F}^{2}}{32 \omega^{4} \hbar^{3}},
$$

where $v_{F} \approx c_{0} / 300$ is the Fermi velocity in graphene. This expression has been derived under the conditions $k_{B} T<$ $\left|\mu_{c}\right|<\hbar \omega$ and the perturbation limits $|\mathbf{E}| \ll \hbar \omega^{2} /\left(e v_{F}\right)$ and $|\mathbf{E}| \ll\left|\mu_{c}\right| \omega /\left(e v_{F}\right)$ for the electric field. For the reference wavelength of $\lambda=1550 \mathrm{~nm}$, Eq. (23) produces a value in the order of $\sigma_{3} \approx-i 10^{-23}\left[\mathrm{~S}(\mathrm{~m} / \mathrm{V})^{2}\right]$.

Another theoretical model for graphene nonlinearity in the optical regime is presented in Ref. 14, providing closedform expressions for two independent elements of the nonlinear surface conductivity tensor $\bar{\sigma}_{s}^{(3)}$, namely, $\sigma_{s, j j k}^{(3)}$ and $\sigma_{s, j k k j}^{(3)} \equiv \sigma_{s, j k j k}^{(3)}$, where $j$ and $k$ are cartesian components tangential to the sheet. The number of nonzero elements of the fourth-rank tensor is eight, just like in the simplest case of Eq. (7) where only one element was required, $\sigma_{3} \equiv \sigma_{s, x x x x}^{(3)}$. At $\lambda=1550 \mathrm{~nm}$ and assuming $\mu_{c}=0.5 \mathrm{eV}$, this model predicts a $\sigma_{3}$ with an imaginary part that is an order of magnitude larger compared to Eq. (23), along with an equally large real part that models TPA in graphene and vanishes for $\mu_{c}>\hbar \omega$. Finally, this model predicts a passage from a selffocusing to a self-defocusing nonlinearity at $\mu_{c} \approx 0.7 \mathrm{eV}$.

Returning to the most general form of $\bar{\sigma}_{s}^{(3)}$ tensor, we can allow for 6 more nonzero elements (total of 14) with 3 of them being independent, while still satisfying the requirement for zero current density normal to the graphene sheet. The additional six elements are $\sigma_{s, j j l}^{(3)}, \sigma_{s, j l j l}^{(3)}$, and $\sigma_{s, j l l j}^{(3)}$, with $j$ denoting the two tangential components and $l$ the normal component. Interestingly, these extra elements imply an interaction of the sheet with the electric field component that is normal to the sheet. Assuming that all six of them are equal to a value in the order of $\sigma_{3} / 3$ (total of two independent elements in the tensor) has been numerically shown to boost the overall nonlinearity of a graphene-clad tapered nanofiber. ${ }^{31}$ However, there is presently no experimental evidence or theoretical model that predicts the magnitude of those elements. The models for the $\bar{\sigma}_{s}^{(3)}$ tensor discussed are recapitulated in Table I.

Finally, an expression for the scalar nonlinear conductivity in the THz regime can be extracted from Ref. 40

$$
\sigma_{3, \mathrm{THz}}=-i \frac{3 e^{4} v_{F}^{2}}{32 \omega^{3} \hbar^{2} \mu_{c}} .
$$

The main difference between this expression and Eq. (23) is the replacement of the photon energy $(\hbar \omega)$ by the chemical potential $\left(\mu_{c}\right)$ in the denominator. At $10 \mathrm{THz}$ and $\mu_{c}=0.3$ $\mathrm{eV}$, the nonlinear surface conductivity is $\sigma_{3} \approx-i 10^{-19}$ $\left[\mathrm{S}(\mathrm{m} / \mathrm{V})^{2}\right]$, that is, more than three orders of magnitude larger than the optical regime. This can lead to very high nonlinear parameter values, in conjunction with the plasmonic confinement exhibited in the $\mathrm{THz}$ band, as discussed in the end of Section III A.

\section{Assessing graphene nonlinearity}

The technologically mastered materials with the highest nonlinear index $\left(n_{2}\right)$ in the optical band include semiconductors (silicon, ${ }^{20} 2.5 \times 10^{-18} \mathrm{~m}^{2} / \mathrm{W}$ or gallium arsenide, ${ }^{41} 10^{-17} \mathrm{~m}^{2} / \mathrm{W}$ ), inorganic glasses (chalcogenides, ${ }^{42}$ $10^{-17} \mathrm{~m}^{2} / \mathrm{W}$ ), and organic polymers (DDMEBT, ${ }^{43}$ $\left.1.7 \times 10^{-17} \mathrm{~m}^{2} / \mathrm{W}\right)$. The real part of $n_{2}$ is typically considered beneficial, whereas the imaginary part constitutes a nonlinear loss mechanism and is thus undesirable; ideally, one would require $\operatorname{Re}\left\{n_{2}\right\} \gg \operatorname{Im}\left\{n_{2}\right\}$, but in this work, we will only focus on the real part of $n_{2}$ as a metric of material nonlinearity.

In order to preliminarily assess graphene's potential for nonlinear applications in the optical regime, we would need to compare its nonlinearity to that of the aforementioned bulk materials, by attributing an equivalent bulk nonlinear index $\left(n_{2, \mathrm{eq}}\right)$ to graphene, ${ }^{15,44}$ according to Eq. (9). Alternatively, one can assess the value of graphene's equivalent nonlinear index without direct knowledge of the nonlinear surface conductivity $\sigma_{s}^{(3)}$. For instance, Ref. 15 estimates $n_{2 \text {,eq }}$ via the saturation intensity assuming the intensity-dependent equivalent susceptibility $\chi_{\mathrm{eq}}^{(1)}(I)=\chi_{\mathrm{eq}}^{(1)} /\left(1+I / I_{\mathrm{sat}}\right)$, where $\chi_{\mathrm{eq}}^{(1)}=i \sigma_{c} /\left(\omega \varepsilon_{0} d_{\mathrm{gr}}\right)$ is a function of the scalar linear surface conductivity $\sigma_{c}$, as in Eq. (8). Furthermore, assuming low intensities and using a first-order Taylor expansion for $\chi_{\mathrm{eq}}^{(1)}(I)$, the

TABLE I. Number of elements in graphene's nonlinear surface conductivity tensor $\bar{\sigma}_{s}^{(3)}$.

\begin{tabular}{lcc}
\hline \hline Source & Nonzero $^{\mathrm{a}}$ & Independent \\
\hline Hexagonal 2D crystal & $8+6$ & $3+3$ \\
Ref. 31 & $8+6$ & $1+1$ \\
Ref. 14 & 8 & 2 \\
Simplest model, Eq. (7) & 8 & 1 \\
\hline
\end{tabular}

assuming the tensor is expressed in a coordinate system that has one cartesian component normal to the graphene sheet. In arbitrary orientations, all 81 elements of the fourth rank tensor can become nonzero, according to Eq. (15).

${ }^{\mathrm{b}}$ Derived from $D_{6 h}$ or $C_{6 v}$ dihedral symmetry groups. ${ }^{26}$ 
equivalent intensity-dependent refractive index of graphene's bulk approximation can be written as $n_{\mathrm{eq}}(I) \approx\left[1+\chi_{\mathrm{eq}}^{(1)}(I)\right]^{0.5}$ $=n_{0, \mathrm{eq}}+n_{2, \mathrm{eq}} I$. In the previous expression, $n_{0, \mathrm{eq}}=\left[1+\chi_{\mathrm{eq}}^{(1)}\right]^{0.5}$ and

$$
n_{2, \mathrm{eq}} \approx-\frac{\chi_{\mathrm{eq}}^{(1)}}{2 I_{\mathrm{sat}} n_{0, \mathrm{eq}}},
$$

where $I_{\text {sat }}=\left|E_{\text {sat }}\right|^{2} /\left(2 Z_{0}\right)$ is the saturation intensity (in units of $\left.\mathrm{W} / \mathrm{m}^{2}\right)$ and $E_{\mathrm{sat}}=2 \omega \mu_{c} /\left(e v_{F}\right)$ is the saturation field strength. It should be noted that Ref. 15 arrives at a slightly different expression, without $n_{0, \mathrm{eq}}$ in the denominator, owing to his initial assumption that $n_{\mathrm{eq}}(I) \approx 0.5 \chi_{\mathrm{eq}}^{(1)}(I)$. Relying on the latter, Ref. 44 includes the full expression of the linear conductivity, with both interband and intraband contributions, in search of an optimized value of $n_{2, \text { eq }}$ with respect to the chemical potential $\mu_{c}$.

Figure 3 presents the $\left|\operatorname{Re}\left\{n_{2, \text { eq }}\right\}\right|$ of graphene as a function of $\mu_{c}$ for various models: The black and blue curves correspond to Eq. (9) using the $\sigma_{s, x x x x}^{(3)}$ derived from the models in Refs. 10 and 14, respectively; the red curve corresponds to Eq. (25) based on the saturation intensity approach. ${ }^{15}$ The solid and dotted parts of the curves correspond to positive and negative values of $\operatorname{Re}\left\{n_{2, \mathrm{eq}}\right\}$, respectively; the notable sign-transitions are attributed to sign transitions of $\operatorname{Im}\left\{\sigma_{c}\right\}$ (Fig. 2(a)), which come into Eq. (9) via $\operatorname{Re}\left\{\varepsilon_{\mathrm{r}, \mathrm{eq}}\right\}$ (Fig. 2(b)). Apparently, the models of Fig. 3 diverge considerably in the entire range of $\mu_{c}$, indicative of the different approximations employed. Nevertheless, we can deduce that the equivalent $n_{2}$ of graphene can be as high as $10^{-15} \mathrm{~m}^{2} / \mathrm{W}$, that is, one or two orders of magnitude larger than the aforementioned bulk nonlinear materials. A recent experimental measurement of graphene nonlinearity using THG from oblique incidence of $789 \mathrm{~nm}$ radiation on a graphene $/ \mathrm{SiO}_{2}$ configuration ${ }^{23}$ revealed a response three orders of magnitude larger compared to bare $\mathrm{SiO}_{2}$; it can be inferred that the equivalent nonlinear index of graphene for THG is $n_{2, \text { eq }}>10^{-17} \mathrm{~m}^{2} / \mathrm{W}$.

Endeavoring to gauge graphene-comprising waveguide nonlinearity, one needs to keep in mind that it is not determined by the properties of its constituting materials $\left(n_{2}\right.$ or $\sigma_{3}$ ) but from its overall nonlinear parameter $\left(\gamma_{\mathrm{NL}}\right)$. In this sense, comparing the nonlinearity of bulk to the equivalent

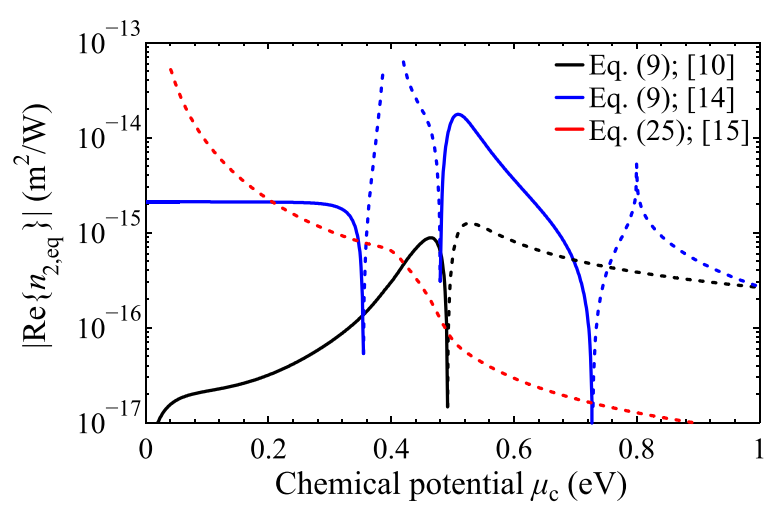

FIG. 3. Absolute value of the real part of the equivalent nonlinear index of graphene, $\left|\operatorname{Re}\left\{n_{2, \text { eq }}\right\}\right|$, as a function of the chemical potential $\mu_{c}$ (Fermi level). The solid and dotted parts of the curves correspond to positive and negative values of $\operatorname{Re}\left\{n_{2, \text { eq }}\right\}$, respectively. one for sheet materials is both deficient and problematic, as it contains a number of pitfalls. The most common mistake is neglecting the anisotropic nature of sheet materials in $3 \mathrm{D}$ waveguides, as was discussed in Section II B, especially for the equivalent nonlinear susceptibility tensor $\bar{\chi}_{\text {eq }}^{(3)}$. For instance, Ref. 44 calculates a giant $\gamma_{\mathrm{NL}}$ for a hybridplasmonic waveguide mode; however, it overlooks the fact that the electric field is predominantly polarized perpendicularly to the graphene sheet and therefore does not interact with it (Eq. (20b)). Another erroneous practice is to calculate the overall $\gamma_{\mathrm{NL}}$ according to the simplified formula $\gamma_{\mathrm{NL}} \approx k_{0} n_{2, \mathrm{eff}} / A$, where $n_{2, \text { eff }}$ might be the nonlinear index of the waveguide core or some spatially averaged variant of it and $A$ might be the effective mode area or the cross-section area of the nonlinear material. In any case, this formula applies only for bulk materials and weakly guiding structures and fails to capture the behavior of ultra-thin sheet materials, such as graphene, used in nanophotonic 3D waveguides. To summarize, we stress that the material nonlinearity itself should always be treated in its natural representation (i.e., as $\bar{\chi}^{(3)}$ for bulk and as $\bar{\sigma}_{s}^{(3)}$ for sheet materials), avoiding "equivalent" representations whenever possible. Moreover, especially in the case of nanophotonic waveguides, rigorous tensorial formulations [e.g., Eqs. (19)] are necessary for the calculation of the nonlinear parameter $\gamma_{\mathrm{NL}}$.

\section{NONLINEAR PARAMETER CALCULATION}

In this section, we will demonstrate the results of our formalism and present them alongside the performance assessment of nonlinear waveguides comprising both sheet and bulk nonlinear materials, such as graphene and silicon, respectively. We consider several optical waveguide archetypes, namely, a silicon wire, a metal stripe, and a metal-insulator-metal (MIM) slot, whose cross sections are depicted in Figs. 4(a)-4(c), respectively. Moreover, we will show how the various pitfalls discussed in Section III C manifest in these waveguide configurations. Finally, we will use the same formalism to calculate the nonlinear parameter of the plasmonic mode supported by a graphene nanoribbon (GNR) waveguide (Fig. 4(d)) operating in the THz band.

In all optical waveguides investigated in this section, the graphene nonlinear surface conductivity, $\sigma_{3, \mathrm{opt}}$, is given by Eqs. (7) and (23). Similarly, its linear surface conductivity, $\sigma_{c}$, is given by Eqs. (21) and (22) for $T=300 \mathrm{~K}, \mu_{c}=0.5$ $\mathrm{eV}, \tau_{1}=10 \mathrm{fs}$, and $\tau_{2}=1.2 \mathrm{ps}$, at $\lambda=1550 \mathrm{~nm}$. In this wavelength range, $\operatorname{Re}\left\{\sigma_{c}\right\}$ significantly increases when $\left|\mu_{c}\right|<0.4 \mathrm{eV}$ (Fig. 2(a)) owing to the contribution of the interband absorption mechanism that eventually leads to higher propagation losses. One final general remark on all the waveguides investigated, the group velocity dispersion propagation length, attributed to material and waveguide dispersion, was found to be much larger than the propagation length associated with either nonlinear effects or losses and thus will be omitted from our calculations.

First, we examine a typical Si-wire waveguide fabricated on an oxide substrate and overlaid with an infinite graphene sheet normal to the $y$-axis (Fig. 4(a)). Silicon is 

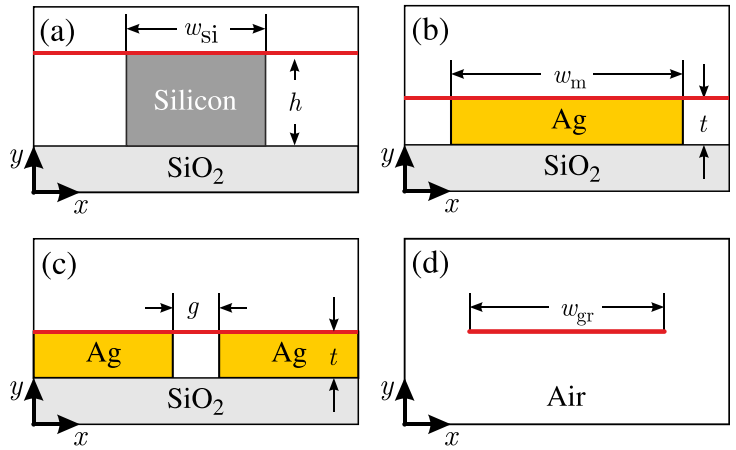

FIG. 4. Nonlinear waveguide configurations investigated. Graphene sheets are depicted by thick red lines and all critical dimensions are annotated. (a) Silicon wire, (b) metal stripe, (c) MIM slot waveguides, overlaid with an infinite graphene sheet, for use in the optical band. (d) Air-suspended GNR waveguide, for use in the $\mathrm{THz}$ band.

characterized by $n_{2, \mathrm{Si}}=2.5 \times 10^{-18} \mathrm{~m}^{2} / \mathrm{W}$ and $n_{0, \mathrm{Si}}=3.48$. Silicon dioxide nonlinearity is neglected and $n_{0, \mathrm{SiO}_{2}}=1.45$. Furthermore, we note that silicon's nonlinear third-order susceptibility tensor, $\bar{\chi}^{(3)}$, is isotropic $(\rho=1)$ and that the only source of losses is $\operatorname{Re}\left\{\sigma_{c}\right\}$.

Employing the procedure described in Section II C, we numerically extract the TE and TM eigenmodes supported by the waveguide and notice that the mode distributions are virtually unaffected by the presence of the graphene sheet. Subsequently, we calculate the nonlinear parameters and propagation losses versus the Si-wire width, while its height is fixed at $340 \mathrm{~nm}$. The results are depicted in Fig. 5 where we note that graphene nonlinearity, $\gamma_{\mathrm{gr}}$, is much lower than the bulk silicon nonlinearity, $\gamma_{\mathrm{Si}}$. It is noteworthy that although graphene does indeed exhibit a large equivalent nonlinear index, this does not necessarily translate into an equally large nonlinear parameter, as witnessed in Fig. 5(a). The misunderstanding usually takes place in the use of $\gamma_{\mathrm{gr}}=k_{0} n_{2, \mathrm{eq}} / A$, or similar formulas, as argued in Section III C: Using the above equation, we mistakenly attribute the huge nonlinear index to the whole area occupied by the mode profile whereas graphene only interacts with a fraction of that area. Another interesting observation, contrary to intuition, is that the TM mode has a larger $\gamma_{\text {gr }}$ than its TE counterpart; the explanation lies with the position of the graphene sheet with respect to the modes' intensity distribution, depicted in the insets of Fig. 5(b). Specifically, on one hand, the TE mode's dominant transverse E-field component, $E_{x}$, is tangential to graphene but only marginally overlaps with it. On the other hand, the TM mode's dominant transverse component, $E_{y}$, is normal to the sheet but it also has a relatively large longitudinal component, $E_{z}$, that significantly overlaps with graphene. Finally, we note that the higher $\gamma_{\text {gr }}$ goes hand-in-hand with higher losses; for comparison, typical losses in fabricated Si-wire waveguides are in the order of $1 \mathrm{~dB} / \mathrm{cm}$ owing to the surface roughness.

To increase $\gamma_{\mathrm{gr}}$, one would either increase graphene nonlinear conductivity, if possible, or attempt to engineer the waveguide configuration. In Fig. 5, while we have chosen a TE mode so as to have the dominant electric field component tangential to graphene, the graphene sheet is placed outside
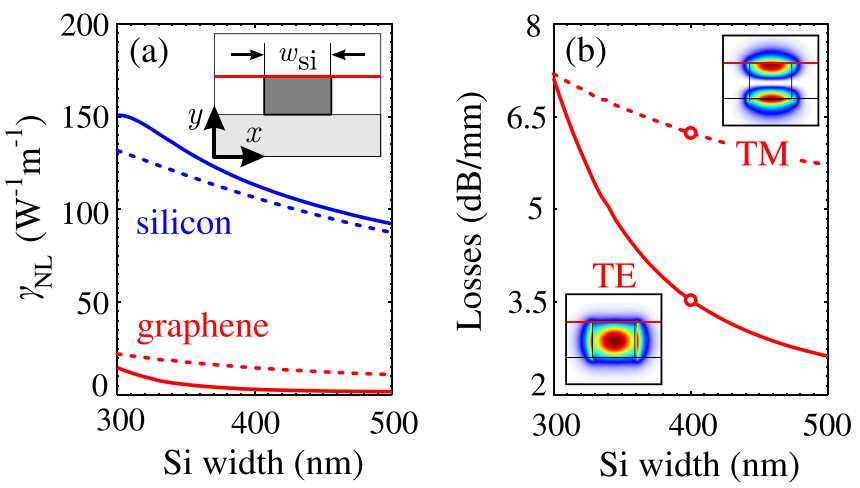

FIG. 5. (a) Numerically calculated nonlinear parameters for a $340 \mathrm{~nm}$ thick $\mathrm{Si}$-wire waveguide overlaid by a graphene sheet, as a function of the Si-wire width. The contributions of silicon and graphene nonlinearity, $\gamma_{\mathrm{Si}}$ and $\gamma_{\mathrm{Si}}$, respectively, are distinguished. Solid and dotted curves correspond to TE and TM modes, respectively. (b) Linear propagation losses owing to graphene conductivity as a function of the Si-wire width. The insets show the TE and TM mode intensity profiles for the electric field components tangential to the graphene sheet, $I_{\tan }=\left|e_{x}\right|^{2}+\left|e_{z}\right|^{2}$, for the Si-width of $400 \mathrm{~nm}$.

the area where the tangential electric field is maximized. To provide further insight in this optimization design, we now consider a 400-nm-wide and 340-nm-thick Si-wire waveguide where the graphene sheet is artificially offset and moved within the silicon core, as shown in the inset of Fig. 6(a). As expected, for the TE mode, graphene nonlinearity contribution becomes comparable to bulk-silicon contribution, $\gamma_{\mathrm{Si}} \sim 110 \mathrm{~W}^{-1} \mathrm{~m}^{-1}$, when the sheet is placed close to the middle of the waveguide. However, this configuration may not be easily fabricated and it also exhibits increased losses (Fig. 6(b)).

Moving on to plasmonic structures, we will now focus on the metal stripe and MIM waveguides depicted in Figs. 4(b) and 4(c), respectively. In both cases, the metal film is $20 \mathrm{~nm}$ thick silver characterized by $n_{0, \mathrm{Ag}}=0.145+11.4 i$ and negligible nonlinearity at $\lambda=1550 \mathrm{~nm},{ }^{22}$ whereas the rest of the material properties are the same as in the previous structure. The metal stripe waveguide is chosen as an archetypical plasmonic waveguide, where we stress that the plasmonic mode we used has a TM polarization and is asymmetric along the $y$-axis. This is not to be confused with
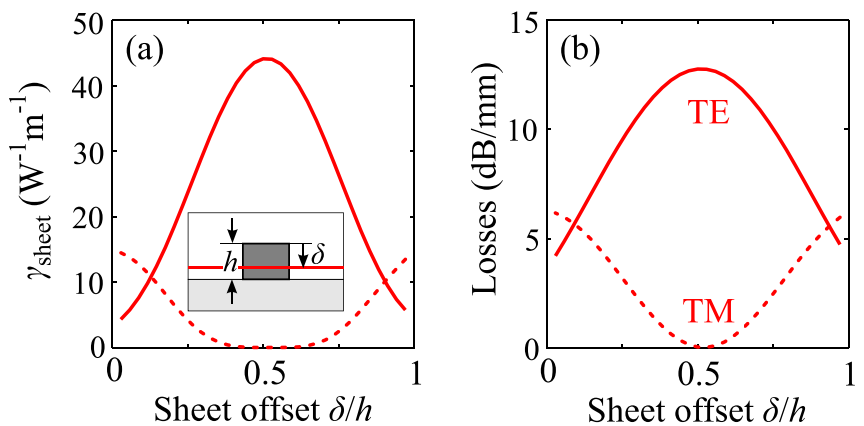

FIG. 6. Numerically calculated (a) sheet nonlinear parameter $\gamma_{\text {sheet }}$ and (b) linear propagation losses, for a $h=340$-nm-thick and 400-nm-wide Si-wire waveguide, as a function of the normalized offset of the graphene sheet from the wire's top side. Solid and dotted curves correspond to TE and TM modes, respectively. The bulk nonlinearity is virtually unperturbed by the location of the graphene sheet, $\gamma_{\text {bulk }} \approx 110 \mathrm{~m}^{-1} \mathrm{~W}^{-1}$. 
the more commonly employed symmetric "long range" TM mode, also supported by the stripe; we verified that the latter has small potential for nonlinear applications due to its very poor confinement. The MIM waveguide structure takes advantage of the metal edge modes; to further elaborate, there are two reasons for choosing a MIM configuration: First, the mode is forced into a TE polarization by the slot geometry, thus ensuring that the dominant electric field component is tangential to the graphene sheet and, second, the metallic interface ensures high confinement as the metal film thickness decreases.

The numerically calculated nonlinear parameter and propagation losses for the metal stripe waveguide are presented in Fig. 7(a). We note that the nonlinear parameter is larger than the Si-wire configuration, and we affirmed that it can be further increased by reducing the metal stripe thickness below $20 \mathrm{~nm}$. The opposite holds for the propagation length, $L_{\text {prop}}$, which poses a limit to the overall nonlinear performance of the metal stripe. Finally, graphene losses at $\mu_{c}=0.5 \mathrm{eV}$ were found to be negligible compared to silver losses: switching off the silver losses results in a tenfold increase to the mode's $L_{\text {prop }}$. The numerical study of the MIM slot configuration is presented in Fig. 7(b). Lowering the waveguide dimensions improves the mode confinement and consequently enhances the nonlinear parameter, as is shown in Fig. 7(a), and we affirmed that the same holds for smaller slot gaps. In fact, the evaluated nonlinear parameter can be six orders of magnitude larger than in the Si-wire case but again with much larger losses. For low slot gaps, the losses are equally due to graphene and bulk-metal conductivity, whereas for larger slot gaps, the losses are mainly due to the bulk-metal, as in the stripe case. The large increase of the nonlinear parameter is mainly attributed to the high field confinement. Moreover, as the main contribution to the nonlinear parameter stems from the overlap of the graphene sheet with the mode within the slot, the nonlinear parameter is especially sensitive to the slot gap. Further optimizing the design, the metal film thickness should be as small as possible in order to squeeze the mode close to the graphene sheet. Finally, replacing the MIM with a
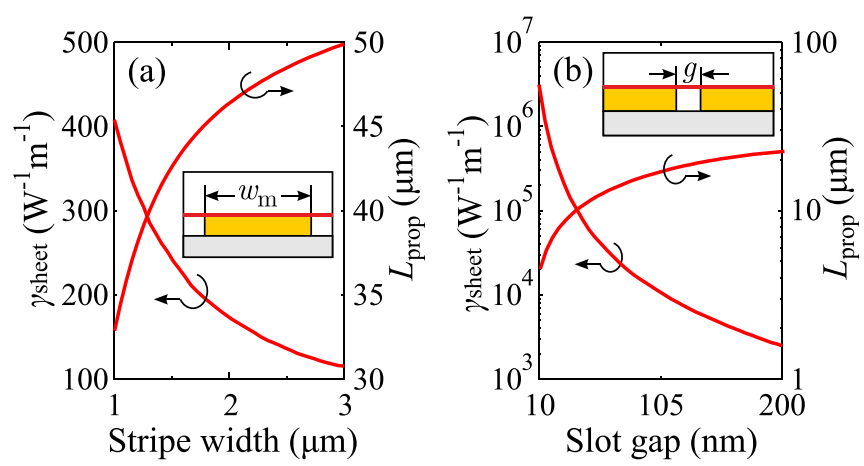

FIG. 7. Numerically calculated sheet nonlinear parameter $\gamma_{\text {sheet }}$ and propagation length $L_{\text {prop }}$ for the highly confined (a) asymmetric TM mode supported by the metal stripe waveguide as a function of the stripe width and (b) TE mode supported by the MIM slot waveguide as a function of the slot gap. The metal in both plasmonic waveguides is a $20 \mathrm{~nm}$ thick silver film, and it is overlaid with an infinite graphene sheet. The $L_{\text {prop }}$ curves include the contribution of both bulk-silver and graphene-sheet conductivities. silicon slot structure would be beneficial to the overall losses, as we dispose of the bulk conductor, but it would not lead to equally high nonlinear parameters; this is because the silicon refractive index is not high enough to retain the same level of confinement that is exhibited by the MIM slot waveguide for a $20 \mathrm{~nm}$ thickness. For the same reasons, hybrid silicon plasmonic structures also exhibit lower performance in terms of the $\mathcal{F}=\gamma_{\mathrm{NL}} L_{\text {prop }}$ nonlinear figure of merit. ${ }^{22}$

The considerably higher value of $\gamma_{\text {sheet }}$ in plasmonic waveguides compared to dielectric ones is due to the extreme field confinement on metal/dielectric interfaces, which is a characteristic of all SPP waveguides; this confinement is especially pronounced near corners or acute features of the metal. Given that graphene is placed in the vicinity of these interfaces, it interacts strongly with the Efield of the waveguide and gives rise to high $\gamma_{\text {sheet }}$. On the contrary, all-dielectric waveguides such as the Si-wire cannot reach the levels of confinement offered by plasmonic structures due to the comparatively limited index-contrast, that is, $\varepsilon_{\mathrm{r}}=3.48^{2}$ vs. 1 (for Si-wires) compared to $\operatorname{Re}\left\{\varepsilon_{\mathrm{r}}\right\}$ $=-130 \mathrm{vs}$. 1 (for silver-based plasmonic guides). Hence, although graphene is again placed where the tangential Efield is maximized (Fig. 6), the $\gamma_{\text {sheet }}$ cannot profit as much from the field/graphene overlap implied in Eq. (19b) or (20b). On the negative side, increased confinement in plasmonic waveguides comes together with high losses from the bulk metal, something that eventually limits their potential for nonlinear applications.

Finally, we numerically calculate the nonlinear parameters for the plasmonic mode supported by the air-suspended 1- $\mu$ m-wide GNR waveguide (Fig. 4(d)) in the $10 \mathrm{THz}$ band. Graphene nonlinear conductivity is evaluated through Eq. (24), whereas its linear conductivity is evaluated through the same formulas that we used in the optical regime, but with $\tau_{1}=40 \mathrm{ps}^{45}$ the interband contribution is negligible assuming that the chemical potential is $\mu_{c} \gg 0.5 \hbar \omega \approx 0.02 \mathrm{eV}$. The GNR mode's nonlinear parameter and propagation length as a function of $\mu_{c}$ are presented in Fig. 8. The nature of this mode is similar to the asymmetric TM plasmonic mode studied in Fig. 7(a) and its electric field distribution for $\mu_{c}=0.3 \mathrm{eV}$ is depicted in the three insets; the linear conductivity and effective index are also given. The resulting nonlinear parameter values are excessively large, which is somewhat anticipated owing to the theoretically predicted nonlinear conductivity in the $\mathrm{THz}$ regime that is three orders of magnitude larger than the optical regime. Moreover, the mode supported is extremely confined at the two edges of the ribbon (see insets of Fig. 8), which further enhances the waveguide nonlinearity. Actually, the GNR in the THz regime can be considered as the ultimately thin version of the plasmonic-stripe waveguide, where graphene acts both as the metal and as the nonlinear material. Although a direct comparison with optical waveguide nonlinearity values is not apt, nevertheless the GNR nonlinearity values demonstrate its potential despite the losses that remain considerable; the propagation length is in the order of $10 \lambda$ for $\mu_{c}=0.3 \mathrm{eV}$. On a final interesting note, tuning the chemical potential from 0.1 to $0.5 \mathrm{eV}$, for instance through electrical gating, one can tweak the nonlinearity through almost three orders of 


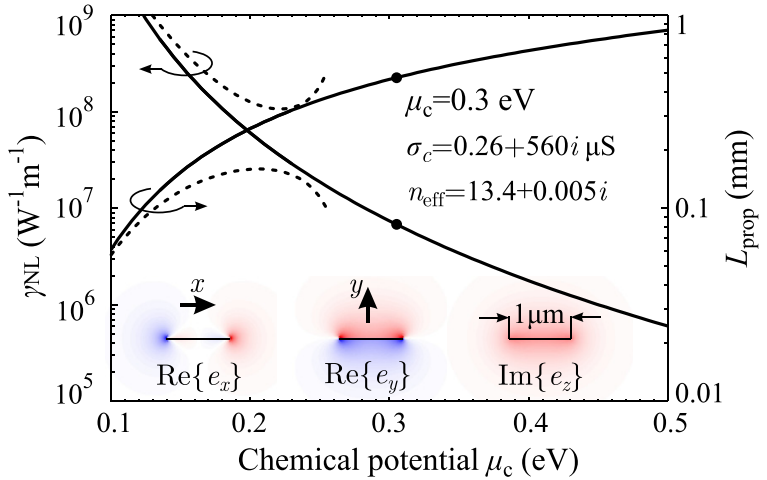

FIG. 8. Numerically calculated nonlinear parameter and propagation length for the plasmonic mode supported by a $1 \mu \mathrm{m}$ wide GNR waveguide at 10 $\mathrm{THz}$, as a function of graphene's chemical potential. The solid curves correspond to the fundamental mode, whose electric field components are depicted in the insets for $\mu_{c}=0.3 \mathrm{eV}$, whereas the dotted curves correspond to the higher order mode that is cut off for $\mu_{c}>0.25 \mathrm{eV}$.

magnitude and at the same time only lose one order of magnitude in the propagation length.

\section{CONCLUSION}

A rigorous electromagnetic modeling for arbitrary crosssection nonlinear nanophotonic waveguides that comprise both bulk and sheet media has been presented. The sheet representation of two-dimensional materials, such as graphene, is superior to the equivalent bulk representation commonly employed, both in terms of consistency with physical properties and computational burden. Utilizing the FEM as a numerical tool and extracting graphene's parameters from theoretical models, we calculated the nonlinear parameter of several types of graphene-comprising waveguides. The performance of these nonlinear waveguides for all-optical switching applications is not remarkable in the optical regime, with the exception of the MIM slot configuration that exhibits a quite large nonlinear parameter and tolerable losses. Nevertheless, convergence between theoretical and experimental results will be required to consolidate those conclusions, as recent experiments ${ }^{12}$ hint at an order of magnitude larger nonlinear surface conductivity for graphene in the optical frequencies, compared to the one predicted by existing theoretical models. Finally, using the same formulation, we identified the promising potential for nonlinear applications in the $\mathrm{THz}$ spectral region exhibited by graphene nanoribbon waveguides.

\section{ACKNOWLEDGMENTS}

This research has been co-financed by the European Union (European Social Fund-ESF) and Greek national funds through the Operational Program "Education and Lifelong Learning" of the National Strategic Reference Framework (NSRF): Research Funding Program THALES (Project ANEMOS). This work was also supported by the "IKY Fellowships of Excellence for Postgraduate Studies in Greece - Siemens Programme".
${ }^{1}$ A. Geim and K. Novoselov, Nature Mater. 6, 183 (2007).

${ }^{2}$ K. Novoselov, V. Fal'Ko, L. Colombo, P. Gellert, M. Schwab, and K. Kim, Nature 490, 192 (2012).

${ }^{3}$ A. N. Grigorenko, M. Polini, and K. S. Novoselov, Nat. Photonics 6, 749 (2012).

${ }^{4}$ S. J. Koester, IEEE J. Sel. Top. Quantum Electron. 20, 84 (2014).

${ }^{5}$ A. Vakil and N. Engheta, Science 332, 1291 (2011).

${ }^{6}$ F. Xia, T. Mueller, Y.-M. Lin, A. Valdes-Garcia, and P. Avouris, Nat. Nanotechnol. 4, 839 (2009).

${ }^{7}$ Q. Bao, H. Zhang, B. Wang, Z. Ni, C. H. Y. X. Lim, Y. Wang, D. Y. Tang, and K. P. Loh, Nat. Photonics 5, 411 (2011).

${ }^{8}$ M. Liu, X. Yin, E. Ulin-Avila, B. Geng, T. Zentgraf, L. Ju, F. Wang, and X. Zhang, Nature 474, 64 (2011).

${ }^{9}$ M. Liu, X. Yin, and X. Zhang, Nano Lett. 12, 1482 (2012).

${ }^{10}$ E. Hendry, P. J. Hale, J. Moger, A. K. Savchenko, and S. A. Mikhailov, Phys. Rev. Lett. 105, 097401 (2010).

${ }^{11}$ H. Zhang, S. Virally, Q. Bao, L. K. Ping, S. Massar, N. Godbout, and P. Kockaert, Opt. Lett. 37, 1856 (2012).

${ }^{12}$ Y. Wu, B. Yao, Y. Cheng, Y. Rao, Y. Gong, X. Zhou, B. Wu, and K. S. Chiang, IEEE Photonics Technol. Lett. 26, 249 (2014).

${ }^{13}$ X. Yao and A. Belyanin, Phys. Rev. Lett. 108, 255503 (2012).

${ }^{14}$ J. L. Cheng, N. Vermeulen, and J. E. Sipe, New J. Phys. 16, 053014 (2014).

${ }^{15}$ J. B. Khurgin, Appl. Phys. Lett. 104, 161116 (2014).

${ }^{16}$ F. Xia, H. Wang, D. Xiao, M. Dubey, and A. Ramasubramaniam, Nat. Photon. 8, 899 (2014).

${ }^{17}$ J. Volakis, A. Chatterjee, and L. Kempel, Finite Element Method for Electromagnetics (IEEE Press, 1998).

${ }^{18}$ G. P. Agrawal, Nonlinear Fiber Optics, 4th ed. (Academic Press, USA, 2007).

${ }^{19}$ X. Chen, N. Panoiu, and R. Osgood, IEEE J. Quantum Electron. 42, 160 (2006).

${ }^{20}$ B. A. Daniel and G. P. Agrawal, J. Opt. Soc. Am. B 27, 956 (2010).

${ }^{21}$ S. Afshar Vahid and T. M. Monro, Opt. Express 17, 2298 (2009).

${ }^{22}$ A. Pitilakis and E. E. Kriezis, J. Opt. Soc. Am. B 30, 1954 (2013).

${ }^{23}$ S.-Y. Hong, J. I. Dadap, N. Petrone, P.-C. Yeh, J. Hone, and R. M. Osgood, Phys. Rev. X 3, 021014 (2013).

${ }^{24}$ Q. Lin, O. J. Painter, and G. P. Agrawal, Opt. Express 15, 16604 (2007).

${ }^{25} \mathrm{R}$. Boyd, Nonlinear Optics (Elsevier Science 2008).

${ }^{26}$ The Elements of Nonlinear Optics, edited by P. Butcher and D. Cotter (Cambridge University Press, Great Britain, 1990).

${ }^{27}$ L. Onural, J. Math. Anal. Appl. 322, 18 (2006).

${ }^{28}$ Y. V. Bludov, A. Ferreira, N. M. R. Peres, and M. I. Vasilevskiy, Int. J. Mod. Phys. B 27, 1341001 (2013).

${ }^{29}$ Q. Bao and K. P. Loh, ACS Nano 6, 3677 (2012).

${ }^{30}$ A. Y. Bykov, T. V. Murzina, M. G. Rybin, and E. D. Obraztsova, Phys. Rev. B 85, 121413 (2012)

${ }^{31}$ A. V. Gorbach, A. Marini, and D. Skryabin, Opt. Lett. 38, 5244 (2013).

${ }^{32}$ A. W. Snyder and J. D. Love, Optical Waveguide Theory (Chapman and Hall, USA, 1983).

${ }^{33}$ D. Smirnova and Y. S. Kivshar, Phys. Rev. B 90, 165433 (2014).

${ }^{34}$ G. Hanson, IEEE Trans. Antennas Propag. 56, 747 (2008).

${ }^{35}$ T. Stauber, N. Peres, and A. Geim, Phys. Rev. B 78, 085432 (2008).

${ }^{36}$ L. A. Falkovsky, Phys. Usp. 178, 923 (2008).

${ }^{37}$ M. Jablan, H. Buljan, and M. Soljačić, Phys. Rev. B 80, 245435 (2009).

${ }^{38}$ T. Gu, N. Petrone, J. F. McMillan, A. van der Zande, M. Yu, G. Q. Lo, D. L. Kwong, J. Hone, and C. W. Wong, Nat. Photonics 6, 554 (2012).

${ }^{39}$ T. Otsuji, S. A. B. Tombet, A. Satou, H. Fukidome, M. Suemitsu, E. Sano, V. Popov, M. Ryzhii, and V. Ryzhii, J. Phys. D: Appl. Phys. 45, 303001 (2012).

${ }^{40}$ S. A. Mikhailov and K. Ziegler, J. Phys.: Condens. Matter 20, 384204 (2008).

${ }^{41}$ J. Aitchison, D. Hutchings, J. Kang, G. Stegeman, and A. Villeneuve, IEEE J. Quantum Electron. 33, 341 (1997).

${ }^{42}$ A. Zakery and S. R. Elliott, J. Non-Crystal. Solids 330, 1 (2003).

${ }^{43}$ B. Esembeson, M. L. Scimeca, T. Michinobu, F. Diederich, and I. Biaggio, Adv. Mater. 20, 4584 (2008).

${ }^{44}$ K. J. A. Ooi, L. K. Ang, and D. T. H. Tan, Appl. Phys. Lett. 105, 111110 (2014).

${ }^{45}$ A. Y. Nikitin, F. Guinea, F. J. García-Vidal, and L. Martín-Moreno, Phys. Rev. B 84, 161407 (2011) 